\title{
DYNAMIC VERSUS STATIC APPROACH TO THEORETICAL ANHARMONIC VIBRATIONAL SPECTROSCOPY OF MOLECULAR SPECIES RELEVANT TO ATMOSPHERIC CHEMISTRY: A CASE STUDY OF FORMIC ACID
}

\author{
BOJANA KOTESKA* VERČE MANEVSKA; ANASTAS MISHEV $\ddagger$ AND LJUPČO PEJOV§
}

\begin{abstract}
Vibrational spectra of the two conformers of the free formic acid molecule are computed by two approaches, with a special emphasis on the region of $\mathrm{O}-\mathrm{H}$ stretching modes. The first approach (referred to as a static one) is based on sequential computation of anharmonic O-H stretching vibrational potential and numerical solution of the vibrational Schrödinger equation by the Numerov method. The second approach (referred to as a dynamic one) is based on molecular dynamics (MD) simulations performed within the atom-centered density matrix propagation scheme (ADMP) followed by spectral analysis of the velocity-velocity and dipole moment autocorrelation functions computed from the ADMP MD trajectories. All calculations are carried out within the density functional tight binding (DFTB) formalism. The computed properties are compared to the available experimental data and the advantages of the dynamic versus the static approach are outlined and analyzed in the context of detection of individual and non-covalently bonded molecular species relevant to climate science and atmospheric chemistry.
\end{abstract}

Key words: formic acid, atmospheric chemistry, molecular dynamics, atom-centered density matrix propagation scheme, anharmonic vibrational frequencies, statistical physics simulations, theoretical spectroscopy, density functional tight binding.

AMS subject classifications. 70F99, 82B30, 92E10

1. Introduction. In the last decade, we have evidenced a thorough paradigmatic shift in many scientific areas, with a notable emphasis on fundamental (i.e. molecular-level) understanding of a wide variety of processes. Within the climate science community in particular, it has become clear that for a thorough in-depth understanding of various phenomena and processes taking place in the Earth's atmosphere, it is necessary to view this complex system at molecular level. Thus, the mesoscale-level models are effectively being replaced by more general molecular models. This, on the other hand, has led to the establishment of chemistry-climate models at various level of sophistication. Numerous molecular species are present in the Earth's atmosphere. However, from the viewpoint of both biosphere and atmospheric chemistry, organic acids are especially important. The title compound studied in the present paper formic acid is the simplest organic acid. It is in a sense a prototypical molecular system for this whole class of compounds. At the same time, it is the most abundant (and ubiquitous) organic acid in the atmosphere $[1,2,3]$. It is generated both by anthropogenic as well as biogenic factors and it is widespread in aerosols and also in atmospheric precipitates (acid rain in particular). Aside from the Earth's atmosphere, formic acid is a prototypical "astrophysical molecule" as well. Numerous studies have been devoted to understanding of the physical and chemical processes that govern its formation in interstellar and cometary ices $[3,4,5,6,7,8,9,10]$.

From a purely fundamental aspect, the formic acid molecule is a prototypical molecular system exhibiting two rotameric forms due to intramolecular hindered rotational motion around the single C-O bond. Existence of two conformational isomers in this simple molecule significantly enriches the spectral appearance in the region where bands due to the $\mathrm{O}-\mathrm{H}$ stretching modes are expected to appear in the vibrational (IR, Raman, inelastic neutron scattering etc.) spectra of gaseous formic acid, as well as in certain noncovalently bonded dimers thereof [1-10]. This spectral region is rather characteristic for detection of noncovalent interactions in which formic acid takes part. Therefore, understanding of the exact shape of this spectral region in the case of free formic acid is of essential importance for further analysis of its appearance in more complex clusters in which it participates. Vibrational modes that involve motion of hydrogen atoms are known to be strongly

*Faculty of Computer Science and Engineering, "Ss. Cyril and Methodious University", Rugjer Boskovikj 16, 1000 Skopje, Republic of Macedonia (bojana.koteska@finki.ukim.mk).

${ }^{\dagger}$ Institute of Chemistry, Faculty of Science, "Ss. Cyril and Methodius University", P.O. Box 162, 1001 Skopje, Republic of Macedonia.

${ }^{\ddagger}$ Faculty of Computer Science and Engineering, "Ss. Cyril and Methodious University", Rugjer Boskovikj 16, 1000 Skopje, Republic of Macedonia (anastas.mishev@finki.ukim.mk).

$\S$ Institute of Chemistry, Faculty of Science, "Ss. Cyril and Methodius University", P.O. Box 162, 1001 Skopje, Republic of Macedonia(1jupcop@pmf.ukim.mk). 
anharmonic. For accurate prediction of the $\mathrm{X}-\mathrm{H}$ stretching vibrational frequencies, therefore, it is crucial to go beyond the often employed harmonic approximation. Further, all the routine computations with widely used quantum chemical codes (either in harmonic approximation, or using an anharmonic approach e.g. by perturbation theoretical treatment [11]) give results that refer to $0 \mathrm{~K}$. Most of the experimental data, however, have been collected at temperatures significantly higher than absolute zero. Having in mind that intramolecular torsional motions can be thermally activated, it is interesting to establish a computationally feasible approach in which the temperature effects could be explicitly accounted for.

Continuing our previous work in the field $[12,13]$, in the present study we tackle both of the previously mentioned issues. We compare the performances of static and dynamics methodologies for computation of anharmonic $\mathrm{O}-\mathrm{H}$ stretching frequencies of the two rotameric forms of free formic acid molecule. The static approach is based on computation of anharmonic O-H stretching vibrational potentials of both conformers followed by numerical solution of the vibrational Schrödinger equation. The dynamic approach, on the other hand, is based on sequential molecular dynamic simulation employing the atom-centered density matrix propagation scheme (ADMP [14]) followed by analysis of several autocorrelation functions computed from the MD trajectories [15].

\section{Computational details.}

2.1. General theoretical methodology and calculation of anharmonic O-H stretching vibrational frequencies. All calculations carried out for the purpose of the present study were performed with the Density functional tight binding methodology (DFTB $[16,17]$ ). The so-called DFTB-A variant of this theoretical approach was used, which is based on usage of analytic form of the relevant matrix elements. The potential energy surface (PES) of free formic acid was thoroughly investigated, employing Schlegel's gradient optimization algorithm [18]. The two minima on the PES, corresponding to the cis- and trans-rotameric forms of this molecular system were located and further characterized. The true-minimum character of the located stationary points on the studied PES was proven by harmonic vibrational analysis performed subsequently to geometry optimization. Absence of negative eigenvalues of the Hessian matrices confirmed that true minima are in question.

To compute the anharmonic $\mathrm{O}-\mathrm{H}$ stretching vibrational frequencies of the two rotamers of formic acid molecule, we have relied on an implementation of a localized mode approach. We start from the equilibrium geometries (corresponding to the minima on the considered PESs) and generate a series of configurations by moving simultaneously the oxygen and hydrogen atoms in a manner that resembles the realistic motion taking place during the excitation of the $\mathrm{O}-\mathrm{H}$ stretching vibration (i.e. keeping the center-of-mass of the $\mathrm{O}-\mathrm{H}$ bond fixed). In the course of these movements, the $\mathrm{O}-\mathrm{H}$ distances were varied from 0.85 to $1.55 \AA$. To generate these configurations, we have used our in-house developed FORTRAN code. A series of 15 single-point energy calculations were further carried out for the series of geometries generated this way to compute the vibrational potentials $V\left(r_{\mathrm{OH}}\right)$. Subsequently, the resulting vibrational Schrödinger equation was solved by the finitedifference Numerov method [12,13]. Frequencies of the fundamental $|0>\rightarrow| 1>$ vibrational transitions were calculated from the energies of the ground $(\mid 0>)$ and first excited $(\mid 1>)$ vibrational energy levels.

2.2. Density functional tight binding (DFTB) molecular dynamics simulations with the atomcentered density matrix propagation (ADMP) scheme. To account explicitly for the finite-temperature effects on molecular structure and dynamics, we have carried out molecular dynamics simulations of free formic acid molecule by the atom-centered density matrix propagation (ADMP) scheme. These simulations were carried out on the basis of forces and energies computed with the DFTB approach, as described in the previous section. The particular MD method that has been implemented in the present study belongs to the extended Lagrangian approaches to molecular dynamics. It is actually based on propagation of the density matrix, using Gaussian-type basis functions $[14,19,20]$. The extended Lagrangian of the studied system is written in the form:

$$
L=\frac{1}{2} \operatorname{Tr}\left(V^{T} M V\right)+\frac{1}{2} \mu \operatorname{Tr}(W W)-E(R, P)-\operatorname{Tr}[\Lambda(P P-P)]
$$

In (2.1), $M, R$ and $V$ are the nuclear masses, positions and velocities, respectively, while $P, W$ and $\mu$ denote the density matrix, density matrix velocity and the fictitious mass for the electronic degrees of freedom, 
correspondingly. $\Lambda$ is a Lagrangian multiplier matrix, and is here used to impose the constraints on the total number of electrons in the system and on the condition of idempotency of the density matrix. If one applies the principle of stationary action, the Euler-Lagrange equations for density matrix propagation can be written in the form:

$$
\begin{gathered}
\mu \frac{d^{2} P}{d t^{2}}=-\left[\left.\frac{\partial E(R, P)}{\partial P}\right|_{R}+\Lambda P+P \Lambda-\Lambda\right] \\
M \frac{d^{2} R}{d t^{2}}=-\left.\frac{\partial E(R, P)}{\partial R}\right|_{P}
\end{gathered}
$$

Throughout the present study, we have used the velocity Verlet algorithm to integrate numerically equations (2.2) and (2.3). The time-evolution of the density matrix (i.e. its propagation) is given by:

$$
\begin{gathered}
P_{i+1}=P_{i}+W_{i} \Delta t-\frac{\Delta t^{2}}{2 \mu}\left[\left.\frac{\partial E\left(R_{i}, P_{i}\right)}{\partial P}\right|_{R}+\Lambda_{i} P_{i}+P_{i} \Lambda_{i}-\Lambda_{i}\right] \\
W_{i+1 / 2}=W_{i}-\frac{\Delta t}{2 \mu}\left[\left.\frac{\partial E\left(R_{i}, P_{i}\right)}{\partial P}\right|_{R}+\Lambda_{i} P_{i}+P_{i} \Lambda_{i}-\Lambda_{i}\right]=\frac{P_{i+1}-P_{i}}{\Delta t} \\
W_{i+1}=W_{i+1 / 2}-\frac{\Delta t}{2 \mu}\left[\left.\frac{\partial E\left(R_{i+1}, P_{i+1}\right)}{\partial P}\right|_{R}+\Lambda_{i+1} P_{i+1}+P_{i+1} \Lambda_{i+1}-\Lambda_{i+1}\right]
\end{gathered}
$$

Within the approach based on extended Lagrangian molecular dynamics with ab initio or semiempirical quantum mechanical Hamiltonian, the electronic subsystem is not treated by a full solution (e.g. by a selfconsistent field procedure). It is propagated along with the nuclear degrees of freedom which are treated classically. To achieve this aim, the time scales of the electronic and nuclear motions are properly adjusted.

Two series of ADMP simulations in the present study were started from the geometries corresponding to the two minima on the DFTB PES of free formic acid, i.e. to the cis- and trans-conformers of this molecular system. Starting from each of the located minima on the DFTB PES, ADMP molecular dynamics simulations have been performed in the microca-nonical $(N V E)$ ensemble. To reach the finally desired temperatures, various amounts of initial nuclear kinetic energies were in the beginning injected into the system and distributed among the atoms. No thermostats were applied to maintain a constant temperature during each of the ADMP simulations. Such approach has led to acceptable temperature fluctuations throughout the simulation. As the main focus of the present study is to compute the spectroscopic properties of the title system within the dynamical approach, i.e. within the time correlation function approach, the dynamics of molecular system has to be sampled properly. In such context, introducing a thermostat to maintain constant temperature, although leading to much smaller temperature fluctuations, would in parallel severely distort the system's dynamics [19, 20]. Series of ADMP simulations were carried out at target temperatures of $10 \mathrm{~K}, 100 \mathrm{~K}, 200 \mathrm{~K}$ and at $300 \mathrm{~K}$.

Upon initial velocity assignment, the system was allowed to equilibrate for 2 ps. Equilibration phase was followed by production (simulation) phase which was 11 ps long. To integrate the equations of motions, a time step of 0.1 fs was used for productive computations. The fictitious electron mass was set to 0.1 amu and the Cholesky basis for the orthonormal set was used.

All static and dynamics calculations in the present study were performed with the Gaussian09 series of codes [21]. Computation of the autocorrelation functions and their subsequent analyses, as well as generation of series of geometries for the pointwise energy calculations were done with our locally developed FORTRAN codes and LINUX scripts.

2.3. Time-correlation functions approach to spectroscopic properties. In the present study, to compute the finite-temperature vibrational spectra of cis- and trans-conformers of free formic acid from the ADMP molecular dynamics simulations, we have implemented the time correlation functions approach. This 
approach is actually based on the linear response formalism [15]. A particular autocorrelation function is computed from the collected data throughout the MD trajectory and it is sequentially Fourier-transformed to arrive at a spectrum of a given type. The autocorrelation of a time-dependent function $\mathrm{f}(\mathrm{t})$, according to Wiener-Khintchine theorem, is given by:

$$
\langle f(\tau) f(t+\tau)\rangle_{\tau}=\frac{1}{2 \pi} \int\left|\int f(t) e^{-i \omega t} d t\right| e^{i \omega t} d \omega
$$

In accordance with the mathematical properties of Fourier transforms, it follows that the autocorrelation of $f(t)$ can be obtained by first taking the Fourier transform of $f(t)$ and sequentially computing the square of its modulus and taking the inverse Fourier transform.

In the present study, we have relied on two types of autocorrelation functions to compute the vibrational spectra: autocorrelation function of the nuclear velocities (the velocity-velocity autocorrelation function) and the dipole moment autocorrelation function [19, 20].

We have used the following definition of the velocity-velocity autocorrelation function (VV-ACF):

$$
\langle\vec{v}(t) \vec{v}(0)\rangle=\sum_{i} \sum_{j} \int_{0}^{T_{l a g}} v_{i, j}\left(t^{\prime}\right) \cdot v_{i, j}\left(t^{\prime}+t\right) d t
$$

where $i$ ranges from 1 to the total number of atoms, while the index $j$ refers to the three principal Cartesian directions and ranges from 1 to 3 . VV-ACF was computed from the data collected from the production (simulation) part of the ADMP trajectory and subsequently normalized with respect to the initial value $\langle\vec{v}(0) \vec{v}(0)\rangle$. From the normalized VV-ACF, the rovibrational density of states spectra, which are proportional to the kinetic energy spectra were computed by:

$$
I_{v v}(\omega)=\lim _{T \rightarrow \infty} \int_{0}^{T} \frac{\langle\vec{v}(0) \vec{v}(0)\rangle}{\langle\vec{v}(0) \vec{v}(0)\rangle} e^{-i \omega t} d t
$$

Analogously to VV-ACF the dipole moment autocorrelation function (DM-ACF) was also computed from the production phase of the ADMP simulation. Infrared absorption cross-sections were computed by a subsequent Fourier transformation, i.e.:

$$
I_{\mu \mu}(\omega) \sim \lim _{T \rightarrow \infty} \int_{0}^{T} \frac{\langle\vec{\mu}(0) \vec{\mu}(0)\rangle}{\langle\vec{\mu}(0) \vec{\mu}(0)\rangle} e^{-i \omega t} d t
$$

To compute the spectra of given types, both types of autocorrelation functions were subjected to Fourier transformation by the fast Fourier transform algorithm (FFT). As the corresponding time series have been obtained from finite length ADMP simulations, we have used the Blackman's window function to account for the fact that $T<\infty$ and cause the integrand to diminish at suitable $T$ values, which (in discrete notation) has the following form [22]:

$$
w(n)=0.42-0.5 \cdot \cos \left(\frac{2 \pi n}{N-1}\right)+0.08 \cdot \cos \left(\frac{4 \pi n}{N-1}\right) ; \quad 0 \leq n \leq N-1
$$

3. Results and discussion. The two minima that have been located on the DFTBA PES of free formic acid molecule, corresponding to the cis- and trans-conformers of this molecular system, are shown in Fig. 3.1. These two structures have further on been used as starting points in the course of MD simulations within the ADMP scheme.

The computed structural parameters for the two minima are compared to the corresponding experimental values in Table 3.1. In this table, also the changes in intramolecular parameters upon mutual interconversion of 

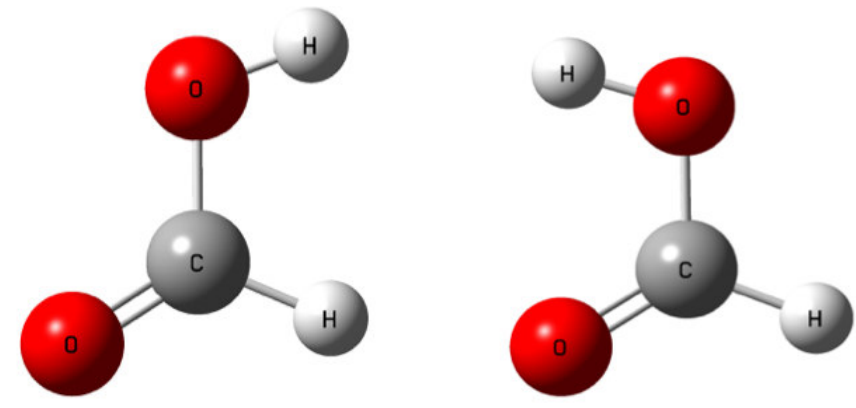

FIG. 3.1. The minima located on the DFTB PES of formic acid molecule, corresponding to the cis- (left) and trans- (right) conformers.

TABLE 3.1

The computed structural parameters for the two minima compared to the corresponding experimental values

\begin{tabular}{|c|c|c|c|c|c|c|}
\hline \multirow[t]{2}{*}{ Parameter } & \multicolumn{2}{|r|}{ Trans } & \multicolumn{2}{|r|}{ Cis } & \multicolumn{2}{|c|}{$\Delta$ (Cis-Trans) } \\
\hline & Exp. & DFTB & Exp. & DFTB & Exp. & DFTB \\
\hline$R_{\mathrm{C}-\mathrm{H}} / \AA$ & 1.097 & 1.135 & 1.105 & 1.153 & 0.008 & 0.018 \\
\hline$R_{\mathrm{C}-\mathrm{O}} / \AA$ & 1.342 & 1.377 & 1.352 & 1.379 & 0.010 & 0.002 \\
\hline$R_{\mathrm{O}-\mathrm{H}} / \AA$ & 0.972 & 0.982 & 0.956 & 0.978 & -0.016 & -0.004 \\
\hline$R_{\mathrm{C}=\mathrm{O}} / \AA$ & 1.203 & 1.211 & 1.195 & 1.202 & -0.008 & -0.009 \\
\hline$\theta \mathrm{HCO}(-\mathrm{H}) /^{\circ}$ & 112.0 & 112.6 & 114.6 & 112.9 & 2.6 & 0.3 \\
\hline$\theta \mathrm{COH} /{ }^{\circ}$ & 106.3 & 107.9 & 109.7 & 110.4 & 3.4 & 2.5 \\
\hline$\theta \mathrm{HCO} /{ }^{\circ}$ & 123.2 & 125.8 & 123.2 & 124.6 & 0.0 & -1.2 \\
\hline$\theta \mathrm{OCO}(-\mathrm{H}) /^{\circ}$ & 124.8 & 121.6 & 122.1 & 122.5 & -2.7 & 0.9 \\
\hline
\end{tabular}

TABLE 3.2

The computed anharmonic $O-H$ and $O-D$ stretching frequencies for the two minima compared to the corresponding experimental values

\begin{tabular}{|l|l|l|l|l|l|l|}
\hline \multirow{2}{*}{ Parameter } & \multicolumn{3}{|r|}{ Trans } & \multicolumn{2}{r|}{ Cis } & \multicolumn{2}{|r|}{$\Delta$ (Cis-Trans) } \\
\cline { 2 - 7 } & Exp. & DFTB & Exp. & DFTB & Exp. & DFTB \\
\hline vO-H / cm-1 & 3550.5 & 3506.0 & 3618.0 & 3557.4 & 67.5 & 51.4 \\
\hline vO-D / cm-1 & 2631.0 & 2569.9 & 2685.0 & 2605.9 & 53.0 & 36.0 \\
\hline
\end{tabular}

the two conformers are given. As can be seen, the overall agreement between theory and experiment is excellent, confirming the appropriateness of the employed theoretical method for the purpose of the present study.

The O-H stretching vibrational potentials, computed at the DFTBA level of theory for the cis-and transconformers are shown in Fig. 3.2. Table 3.2, on the other hand, compiles the computed anharmonic O-H and O-D stretching frequencies for the two minima compared to the corresponding experimental values. As can be seen, concerning the fact that these are the "raw" (i.e. unscaled) values, the agreement between theoretically computed and experimentally measured $\mathrm{O}-\mathrm{H}(\mathrm{D})$ stretching frequencies is rather satisfactory.

In the present study, to carry out the molecular dynamics simulations, we have relied on the atom-centered density matrix propagation scheme. This is an extended Lagrangian molecular dynamics method in which the electronic structure is accounted for by a single-particle density matrix representation of the electronic subsystem. The density matrix is propagated simultaneously with the nuclear degrees of freedom (treated in a classical manner). This is achieved by introducing the fictitious inertia tensor $\mu$ in the course of adjusting the nuclear with the electronic time scales. The overall effect of such adjustment is an achievement of a fictitious dynamics that allows controllable oscillations around the Born-Oppenheimer surface. However, within the ADMP scheme the self-consistent field (SCF) convergence is not achieved. Therefore, one has to analyze 


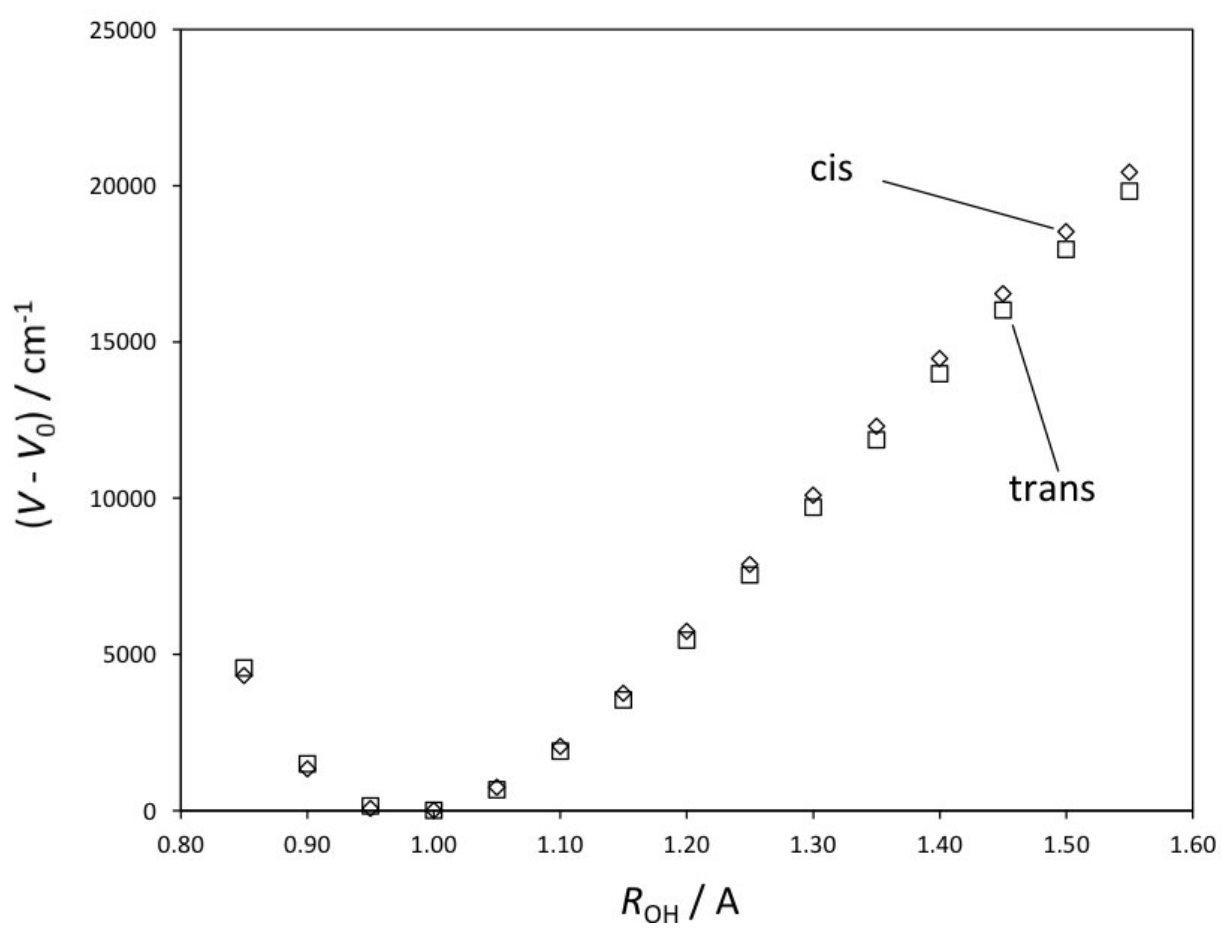

FIG. 3.2. The $O-H$ stretching vibrational potentials for the cis-and trans-conformers of free formic acid, computed at the DFTBA level of theory.

carefully the errors in order to be certain of the accuracy of the dynamics as well as of its physical meaningfulness.

To analyze the errors inherent to the ADMP scheme, in our present study, we have thoroughly analyzed the time-evolution of the adiabaticity index, the idempotency of the density matrix [19, 20], as well as timedependence of the total angular momentum throughout the productive part of the simulation. On Fig. 3.3, the time-dependence of the adiabaticity index in the production phase of the simulations carried out at $10 \mathrm{~K}$, starting from both cis- and trans-conformers of the formic acid molecule is shown. Comparison with the literaturesuggested threshold values of this quantity $[19,20]$ allowed us to conclude the stability of the simulations. As an additional test, to confirm the previous conclusion, we have also checked the idempotency of the density matrix; this parameter was kept within the threshold value of $10^{-12}$. At the same time, the total angular momentum value was conserved to $<10^{-13} \hbar$ as well.

In Table 3.3, the target MD temperatures are compared to the actual ones achieved during the productive part of the ADMP simulation runs with cis- and trans-conformers as starting points for the dynamical simulations (i.e. the minima on the DFTB PESs corresponding to these structures). The observed temperature fluctuations around the target and average values presented in Table 3.3 throughout the productive phase of the simulation were acceptable and, at the same time, in line with the statistical physics expectations for a dynamical simulation of molecular system with the current size. Certainly, more precise temperature control (and therefore, further diminishing of the temperature fluctuations) could have easily been achieved by e.g. coupling the system to a suitably chosen thermostat. However, such temperature control has not been applied in the present study, since the main intention of the study was computation of spectroscopic properties from dynamical simulations through the time correlation functions formalism. In order to do this in a physically correct manner, any distortion of the dynamics, which would be introduced by the imposed temperature control $[19,20]$, has to be avoided.

In Fig. 3.4, the frequency region of the kinetic energy spectra (i.e. the kinetic energy density of states spectra) obtained by Fourier transformation of the velocity-velocity autocorrelation function in which peaks 

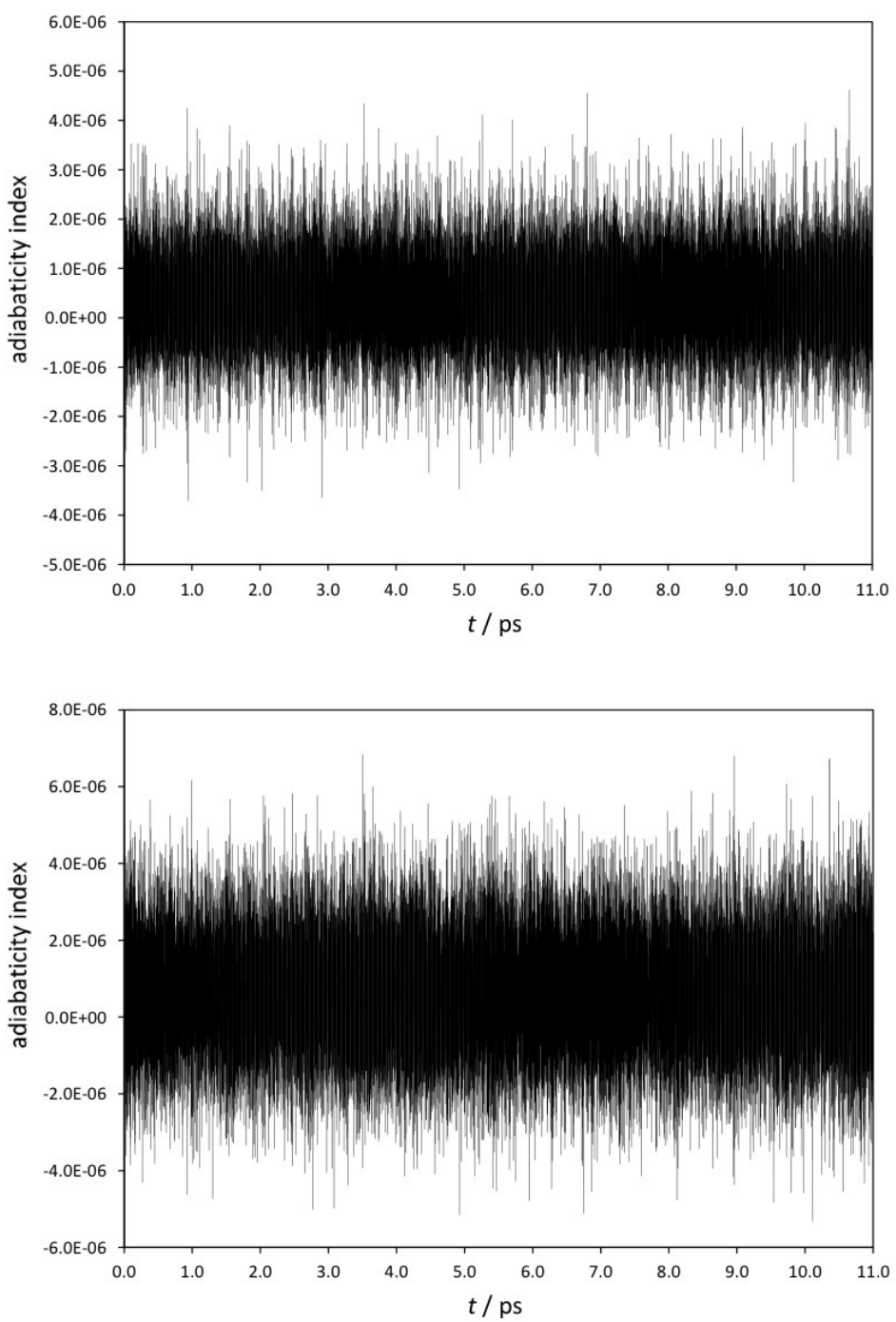

FIG. 3.3. The time-dependence of the adiabaticity index in the production phase of the simulations carried out at $10 \mathrm{~K}$, starting from both cis- (top) and trans-conformers (bottom) of the formic acid molecule.

TABLE 3.3

Target and actual temperatures achieved during the productive part of the ADMP simulation runs with cis- and transconformers as starting points for the dynamical simulations (i.e. the minima on the DFTBA PESs corresponding to these structures)

\begin{tabular}{|l|l|l|}
\hline$T_{\text {target }} / \mathrm{K}$ & $T_{\text {sim.,cis }} / \mathrm{K}$ & $T_{\text {sim.,trans }} / \mathrm{K}$ \\
\hline 10 & 10.15 & 9.97 \\
\hline 100 & 101.91 & 99.59 \\
\hline 200 & 201.97 & 199.90 \\
\hline 300 & 299.72 & 298.45 \\
\hline
\end{tabular}

due to the $\mathrm{O}-\mathrm{H}$ stretching vibrational motions are expected to appear is shown for the series of simulations carried out at the four different temperatures: 10, 100, 200 and $300 \mathrm{~K}$ starting from the equilibrium geometry 


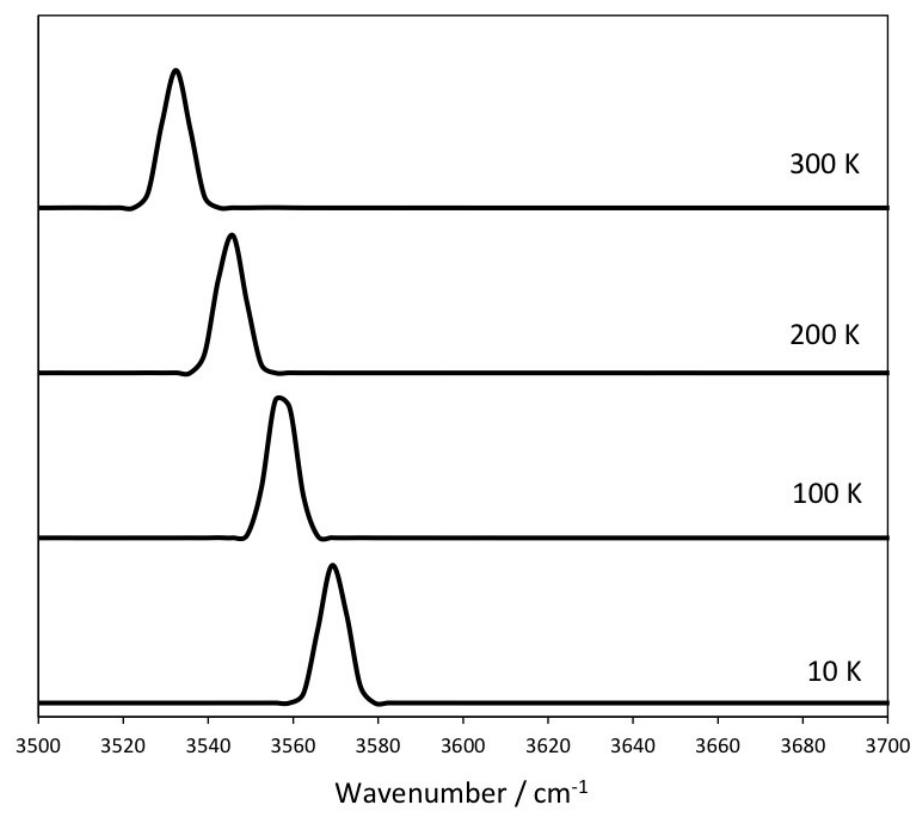

FIG. 3.4. The kinetic energy spectra (kinetic energy density of states spectra) obtained by Fourier transformation of the velocity-velocity autocorrelation function for the series of simulations carried out at the four different temperatures, starting from the trans-conformer of the free formic acid molecule, in the frequency region where signals due to the $O$ - $H$ stretching vibrations are expected to appear (from 3500 to $3700 \mathrm{~cm}^{-1}$ ).

of the trans-conformer of formic acid. In Fig. 3.5, on the other hand, the same spectral region is shown in the kinetic energy spectra computed from the trajectories at the mentioned series of temperatures started from the equilibrium geometry corresponding to the cis-conformer of free formic acid molecule. The intensity pattern in the kinetic energy spectra is, of course, not directly comparable to the infrared spectrum. It is more directly comparable to the deep inelastic neutron scattering spectrum. However, these spectra still contain essential information concerning the spacings between vibrational energy levels of different intramolecular modes. Following, therefore, the kinetic energy spectra, one indirectly follows the temperature evolution of the energy level differences. As will be seen from the subsequent discussion, the particular frequency region analyzed in the present study maps quite clearly the temperature evolution of the molecular conformational flexibility along with the intramolecular vibrational energy redistribution.

In summary, the kinetic energy spectra carry out the essential information concerning molecular rovibrational density of states (or, perhaps even more precisely, about the existence of energy level difference at particular frequency, i.e. wavenumber value). Although for a direct comparison with the experimental infrared spectra, it is better to rely on a straightforwardly comparable quantity derived by a Fourier transformation of the dipole moment autocorrelation function, throughout this present study, we will use the kinetic energy spectra in parallel with those computed from the dipole autocorrelation function. Although this may, at first sight, seem to complicate the analysis, we have chosen such an approach due to the following reasons. Existence of vibrational energy level difference, manifested with an appearance of a band (signal) at a given frequency value, does not tell us anything about the particular mode that is involved in this energy level pattern. As a matter of fact, the intramolecular nuclear motions that correspond to a particular mode may or may not involve a change in the dipole moment. Such change, however, would be responsible for absorption of light quanta upon interaction with the incident radiation from infrared spectral region. By restricting the analysis solely on the basis of spectra obtained from the dipole moment autocorrelation function, therefore, the thermally-induced behavior of modes which are not infrared active would be disregarded.

In Fig. 3.6, the $\mathrm{O}-\mathrm{H}$ stretching region in the spectra computed by Fourier transformation of the dipole moment vector autocorrelation function for the series of simulations of free formic acid molecule carried out 


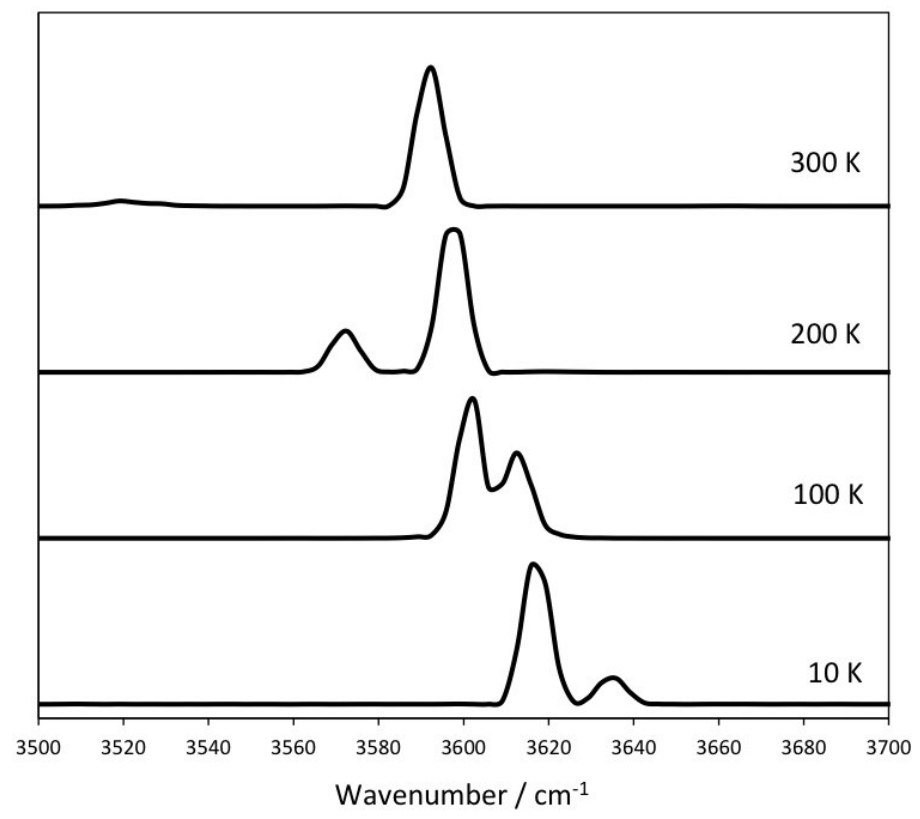

FIG. 3.5. The kinetic energy spectra (kinetic energy density of states spectra) obtained by Fourier transformation of the velocity-velocity autocorrelation function for the series of simulations carried out at the four different temperatures, starting from the cis-conformer of the free formic acid molecule, in the frequency region where signals due to the $O$ - $H$ stretching vibrations are expected to appear (from 3500 to $3700 \mathrm{~cm}^{-1}$ ).

at the four different temperatures $(10,100,200$ and $300 \mathrm{~K})$, started from the equilibrium geometry of the trans-conformer is shown. Fig. 3.7, on the other hand, shows the same spectral region in the dipole moment autocorrelation spectra extracted from series of simulations at the mentioned temperatures, this time started from the equilibrium geometry of the cis-conformer of free formic acid molecule. As implied before, these spectra are actually directly comparable to the experimentally measured frequency dependencies of the infrared absorption cross-sections obtained by experimental infrared spectroscopic techniques, i.e. directly comparable to the temperature-dependent infrared spectra of the studied species.

We start the discussion by analyzing the appearance of the spectral region in which signals due to $\mathrm{O}-\mathrm{H}$ stretching motions are expected to appear in the kinetic energy spectra computed from the velocity-velocity autocorrelation function (Figs. 3.4 and 3.5), corresponding to the rovibrational density of states. As can be seen from Fig. 3.4, the $\mathrm{O}-\mathrm{H}$ stretching band exhibits a notable shift towards lower wavenumbers (energies) upon temperature increase. We attribute this behavior to the following. At lower initial kinetic energies, corresponding to achievement of lower effective temperatures, the amplitudes of all intramolecular motions are modest, much lower than in the case of higher energies (and consequently, temperatures). At lower temperatures, therefore, the $\mathrm{O}-\mathrm{H}$ oscillators sample only the region in the vicinity of the "harmonic part" of the vibrational potential. As the temperature increases, the amplitudes increase, so that the studied oscillators more frequently reside in the "more anharmonic" region of the potential. As the differences between vibrational energy levels are directly dependent on the anharmonicity, the more anharmonic the effective (sampled) potential is, the lower in energy will the corresponding spectral band appear. Since the trans- rotamer of the free formic acid is the more stable one, only a single effective $\mathrm{O}-\mathrm{H}$ stretching band appears at temperatures up to $300 \mathrm{~K}$ in the rovibrational density of states spectra. The situation in the case of simulations started from the cis-form of free formic acid is much more complex, however (Fig. 3.5). Though the general trend in the temperaturedependence of the most intensive band in this spectral region basically follows the one evidenced in the case of trans-conformer, it can be seen from Fig. 3.5 that in the studied spectral region of the cis-conformer, even at temperatures as low as $10 \mathrm{~K}$, two bands appear, as a consequence of enhanced amplitudes of the intramolecular $\mathrm{OCOH}$ torsional motion. At temperature of $200 \mathrm{~K}$, a clearly defined band appears at $\sim 3572 \mathrm{~cm}^{-1}$, which 


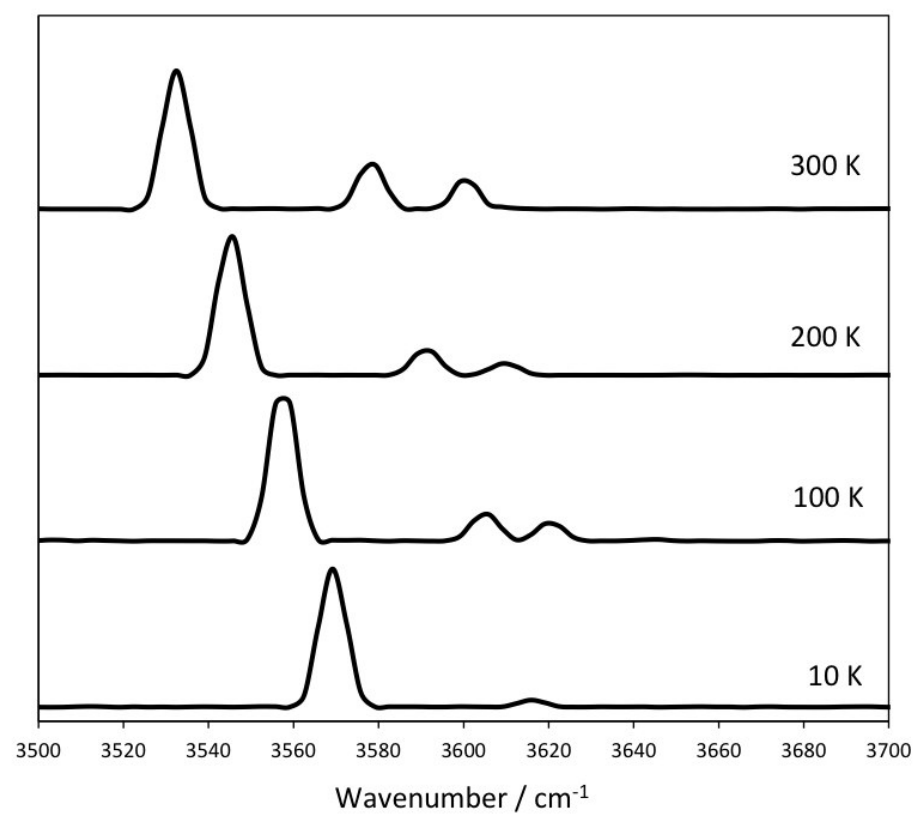

FIG. 3.6. The dipole moment autocorrelation spectra ( infrared absorption spectra) obtained by Fourier transformation of the dipole moment autocorrelation function for the series of simulations carried out at the four different temperatures, starting from the trans-conformer of the free formic acid molecule, in the frequency region where signals due to the $O$ - $H$ stretching vibrations are expected to appear (from 3500 to $3700 \mathrm{~m}^{-1}$ ).

could correspond to an $\mathrm{O}-\mathrm{H}$ stretching vibration within the deeper well of the torsional potential. The velocityvelocity autocorrelation spectra computed from the ADMP simulations in the present study are based on the autocorrelation functions of averaged nuclear velocities. Therefore, these are statistical quantities, obtained as a statistical average from numerous different configurations spanned by the MD simulation and the computed resultant spectrum corresponds to a dynamically averaged picture of the studied molecular system. In the course of dynamical simulation, the immediate surroundings of the $\mathrm{O}-\mathrm{H}$ stretching vibrational mode changes in an anisotropic manner, which, in turn, leads to broadening and flattening of particular spectral regions, in line with previous results reported in the literature $[19,20]$.

The spectral pictures observed in the $\mathrm{O}-\mathrm{H}$ stretching regions of the dipole moment autocorrelation spectra (Fig. 3.6 and 3.7) computed from the trajectories started from trans- and cis- minima on the DFTB PES of free formic acid resemble quite much the analogous regions in the velocity-velocity autocorrelation spectra, with some intensity redistributions.

On the basis of all previously outlined theoretical results one can conclude that the thermally-induced dynamical effects in the rovibrational density of states (as well as in the infrared absorption cross-sections) of even rather simple individual molecular systems may lead to substantial differences in comparison to the corresponding "static" properties. When one compares theoretical with experimental spectroscopic data, therefore, to account for all the complexities inherent to the intramolecular motions of the studied system, the temperature effects should be explicitly accounted for. This is especially valid considering the fact that the usually available experimental vibrational spectroscopic data have often been obtained at temperatures far above the absolute 0 (which is the effective temperature at which "static" ab intio or semiempirical computations are often carried out).

When the studied molecular system is characterized by a certain degree of intramolecular flexibility, i.e. in cases when several rotamers close in energy may exist, thermally-induced intramolecular motions and intramolecular vibrational energy redistributions can cause effective dynamical transitions between the corresponding wells on the molecular PES. As the statistically averaged spectra computed from statistical physics simulations by the linear response formalism account for such dynamical effects (and for the intramolecular conformational 


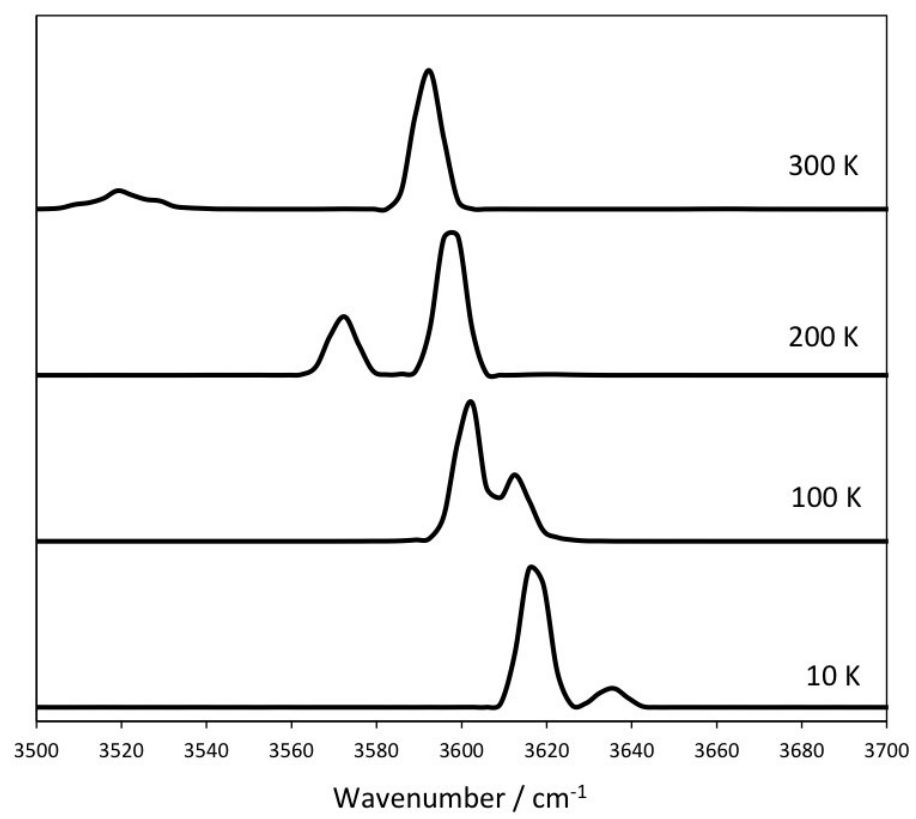

FIG. 3.7. The dipole moment autocorrelation spectra ( infrared absorption spectra) obtained by Fourier transformation of the dipole moment autocorrelation function for the series of simulations carried out at the four different temperatures, starting from the cis-conformer of the free formic acid molecule, in the frequency region where signals due to the O-H stretching vibrations are expected to appear (from 3500 to $3700 \mathrm{~m}^{-1}$ ).

flexibility of the studied molecule as well), these should be favored over the static ones.

4. Summary and conclusions. In the present study, we have carried out a density functional tight binding study of the $\mathrm{O}-\mathrm{H}$ stretching vibrations in the case of two conformers of free formic acid molecule. We have calculated the anharmonic $\mathrm{O}-\mathrm{H}$ stretching vibrational frequencies implementing a static approach (based on calculation of the $\mathrm{O}-\mathrm{H}$ stretching vibrational potentials of the two formic acid conformers and subsequent numerical solution of the vibrational Schrödinger equation), and a dynamic approach (based on sequential DFTB MD simulation within the ADMP scheme followed by analysis of several autocorrelation functions calculated from the MD trajectories). Dynamic calculations allowed us to compute the temperature-dependent spectroscopic properties of this molecule, i.e. to explicitly involve the thermal motion effects in single-molecule computational anharmonic vibrational spectroscopy. We have computed various types of spectra at series of temperatures (ranging from 10 up to $300 \mathrm{~K}$ ), including the rovibrational density of states spectra, as well as the infrared absorp-tion cross section spectra. The thermally induced changes in the single-molecule spectroscopic properties were deduced and the reasons behind them were analyzed and discussed. The advantages of the dynamic approach to computational vibrational spectroscopy at finite temperatures are outlined and discussed, in the context of research devoted to molecular-level understanding of the phenomena and processes relevant to climate science and atmospheric chemistry.

Acknowledgments. This work was supported by the European Unions Horizon 2020 research and innovation programme, project Virtual Research Environment for Regional Interdisciplinary Collaboration in Southeast Europe and Eastern Mediterranean VI-SEEM [675121].

\section{REFERENCES}

[1] D.P. Tew And W. Mizukami. Ab initio vibrational spectroscopy of cis-and trans-formic acid from a global potential energy surface. The Journal of Physical Chemistry A, 120(49):9815-9828, 2016. 
[2] S. Roszak, R.H. Gee, K. Balasubramanian, And L.E. Fried. New theoretical insight into the interactions and properties of formic acid: Development of a quantum-based pair potential for formic acid. The Journal of chemical physics, 123(14):144702, 2005.

[3] M. Pettersson, J. Lundell, L. Khriachtchev, And M. RÄsänen. Ir spectrum of the other rotamer of formic acid, cis-hcooh. Journal of the American Chemical Society, 119(48):11715-11716, 1997.

4] M. Pettersson, E.M.S. MaçôAs, L. Khriachtchev, R. FAusto, And M. RÄsÄnen. Conformational isomerization of formic acid by vibrational excitation at energies below the torsional barrier. Journal of the American Chemical Society, 125(14):4058-4059, 2003.

[5] E.M.S. Macôas, L. Khriachtchev, M. Pettersson, J. Lundell, R. Fausto, And M. RÄsänen. Infrared-induced conformational interconversion in carboxylic acids isolated in low-temperature rare-gas matrices. Vibrational spectroscopy, 34(1):73-82, 2004.

[6] M. Pettersson, E.M.S. MaçôAs, L. Khriachtchev, J. Lundell, R. Fausto, And M. Räsänen. Cis $\rightarrow$ trans conversion of formic acid by dissipative tunneling in solid rare gases: Influence of environment on the tunneling rate. The Journal of chemical physics, 117(20):9095-9098, 2002.

[7] E.M.S. MaÇôAs, L. Khriachtchev, M. Pettersson, J. Juselius, R. FAusto, And M. RÄsÄnen. Reactive vibrational excitation spectroscopy of formic acid in solid argon: Quantum yield for infrared induced trans $\rightarrow$ cis isomerization and solid state effects on the vibrational spectrum. The Journal of chemical physics, 119(22):11765-11772, 2003.

[8] E.M.S. Maçôas, J. Lundell, M. Pettersson, L. Khriachtchev, R. Fausto, and M. Räsänen. Vibrational spectroscopy of cis-and trans-formic acid in solid argon. Journal of Molecular Spectroscopy, 219(1):70-80, 2003.

[9] S. Ioppolo, B.A. McGuire, M.A. Allodi, And G.A. Blake. Thz and mid-ir spectroscopy of interstellar ice analogs: methyl and carboxylic acid groups. Faraday discussions, 168:461-484, 2014.

[10] G. BuEmI. Dft study of the hydrogen bond strength and ir spectra of formic, oxalic, glyoxylic and pyruvic acids in vacuum, acetone and water solution. Journal of Physical Organic Chemistry, 22(10):933-947, 2009.

[11] V. BARONE. Anharmonic vibrational properties by a fully automated second-order perturbative approach. The Journal of chemical physics, 122(1):014108, 2005.

[12] B Koteska, A. Mishev, And L.J. Pejov. Computational study of intramolecular oh stretching vibrations in the two rotamers of free formic acid. Romanian Reports in Physic, in press.

[13] B. Koteska, A. Mishev, And L. Pejov. Computational approach towards vibrational spectroscopic detection of molecular species relevant to atmospheric chemistry and climate science: The formic acid rotamers. In Smart Technologies, IEEE EUROCON 2017-17th International Conference on, pages 926-931. IEEE, 2017.

[14] H.B. Schlegel, J.M. Millam, S.S. Iyengar, G.A. Voth, A.D. Daniels, G.E. Scuseria, And M.J. Frisch. Ab initio molecular dynamics: Propagating the density matrix with gaussian orbitals. The Journal of Chemical Physics, 114(22):9758-9763, 2001.

[15] M. Thomas, M. Brehm, R. Fligg, P. Vöhringer, And B. Kirchner. Computing vibrational spectra from ab initio molecular dynamics. Physical Chemistry Chemical Physics, 15(18):6608-6622, 2013.

[16] M. Elstner, D. Porezag, G. Jungnickel, J. Elsner, M. Haugk, T. Frauenheim, S. Suhai, and G. Seifert. Selfconsistent-charge density-functional tight-binding method for simulations of complex materials properties. Physical Review B, 58(11):7260, 1998 .

[17] G. Zheng, H.A. Witek, P. Bobadova-Parvanova, S. Irle, D.G. Musaev, R. Prabhakar, K. Morokuma, M. Lundberg, M. Elstner, C. KöHLER, ET AL. Parameter calibration of transition-metal elements for the spin-polarized self-consistentcharge density-functional tight-binding (dftb) method: Sc, ti, fe, co, and ni. Journal of chemical theory and computation, 3(4):1349-1367, 2007.

[18] H.B. Schlegel. Optimization of equilibrium geometries and transition structures. Journal of Computational Chemistry, $3(2): 214-218,1982$.

[19] S.S. IYENGAR. Dynamical effects on vibrational and electronic spectra of hydroperoxyl radical water clusters. The Journal of chemical physics, 123(8):084310, 2005.

[20] S.S. Iyengar, M.K. Petersen, T.J.F. Day, C.J. Burnham, V.E. Teige, And G.A. Voth. The properties of ion-water clusters. $i$. the protonated 21-water cluster. The Journal of chemical physics, 123(8):084309, 2005.

[21] MJ Frischet ET AL. al., gaussian 09, revision a. 1, 2009.

[22] F.J. HARRIS. On the use of windows for harmonic analysis with the discrete fourier transform. Proceedings of the IEEE, $66(1): 51-83,1978$.

Edited by: Aneta Karaivanova

Received: Dec 22, 2017

Accepted: Apr 10, 2018 